\title{
Sentidos de la formación bioética de enfermeros en un contexto intercultural
}

\author{
Meaning of the bioethical training of nurses in an intercultural context
}

Sentidos da formação bioética de enfermeiros em um contexto intercultural

\section{Adriana Lucia Valdez Fenández ${ }^{1,2}$}

${ }^{1}$ Enfermera, candidata a doctora en Ciencias de la Educación. Profesora asistente de planta de la Facultad de Ciencias de la Salud, Universidad del Cauca, Colombia.

${ }^{2}$ Este artículo es producto de una investigación realizada en el marco del desarrollo de la tesis doctoral titulada "Sentidos sobre la bioética que emergen en las prácticas formativas de enfermería en un contexto intercultural", presentada por la autora para optar al título de doctora en Ciencias de la Educación de la Universidad del Cauca-Rudecolombia.

Cómo citar este artículo en edición digital: Valdez Fernández, A. (2020). Sentidos de la formación bioética de enfermeros en un contexto intercultural. Cultura de los Cuidados (Edición digital), 24 (57) Recuperado de http://dx.doi.org/10.14198/cuid.2020.57.16

Correspondencia: Departamento de Enfermería, Facultad de Ciencias de la Salud, Universidad del Cauca. Calle $5 \mathrm{~N}^{\circ}$ 4-70 de Popayán (Cauca, Colombia).

Correo electrónico de contacto: adrianitalvf@unicauca.edu.co

\begin{abstract}
Humanizing education constitutes a pillar for bioethical formation of nursing professionals in intercultural contexts, which guides the transformative sense of the vision of disciplinary care. It was sought to understand the senses that the training actors grant bioethics from the training practices in an intercultural context. For this, a descriptive qualitative investigation with a hermeneutical epistemic approach is proposed, the data analysis was carried out from the grounded theory. Data were
\end{abstract}

collected through: documentary review, semi-structured interviews, non-participant observation, field diaries and focus groups. Participants correspond to students, teachers and directors of the nursing program of a university in southwestern Colombia, practice tutors and care subjects who were attended by the students. The results allow us to conclude that the main nonsense in formation lies in a problem of coloniality of being and knowing, because the curriculum continues to privilege technical knowledge over a human formation. Weaving intercultural bioethics from pedagogical reflection in intercultural contexts allows 
developing a critical training regarding what does not favor the harmony of human being with territory and that leads the professional future to be aware of their responsibility with health care.

Keywords: Nursing, care, interculturality, bioethics, education.

\section{RESUMEN}

Humanizar la educación se constituye en un pilar para la formación bioética de los profesionales de enfermería en contextos interculturales, lo que orienta el sentido transformador de la visión de cuidado disciplinar. Con este estudio se buscó comprender los sentidos que otorgan a la bioética los actores de formación desde las prácticas formativas en un contexto intercultural. Para ello se planteó una investigación cualitativa descriptiva con enfoque epistémico hermenéutico; el análisis de datos se realizó desde la teoría fundamentada. Se recolectaron datos a través de: revisión documental, entrevistas semiestructuradas, observación no participante, diarios de campo y grupos focales. Los participantes corresponden a los estudiantes, docentes y directivos del programa de enfermería de una universidad del suroccidente colombiano, tutores de práctica y sujetos de cuidado que fueron atendidos por los estudiantes. Los resultados permitieron concluir que el principal sinsentido en la formación radica en un problema de colonialidad del ser y saber, porque el currículo continúa privilegiando el saber técnico sobre una formación humana. Tejer la bioética intercultural desde la reflexión pedagógica en contextos interculturales permite desarrollar una formación con sentido crítico respecto aquello que no favorece la armonía del ser humano con el territorio y que conlleve al futuro profesional a ser consciente de su responsabilidad con el cuidado de salud.

Palabras clave: Enfermería, cuidado, interculturalidad, bioética, educación.

\section{RESUMO}

A educação humanizada constitui um pilar para a formação bioética dos profissionais de enfermagem em contextos interculturais, que norteiam o sentido transformador da visão do cuidado disciplinar. Buscou-se compreender os sentidos que os atores treinadores conferem bioética a partir das práticas de treinamento em um contexto intercultural. Para isso, propõe-se uma investigação qualitativa descritiva, com abordagem epistêmica hermenêutica, e a análise dos dados foi realizada a partir da teoria fundamentada. Os dados foram coletados por meio de: revisão documental, entrevistas semiestruturadas, observação não participante, diários de campo e grupos focais. Os participantes correspondem a estudantes, professores e diretores do programa de enfermagem de uma universidade no sudoeste da Colômbia, tutores de prática e assuntos de atendimento que foram atendidos pelos alunos. Os resultados permitem concluir que o principal absurdo da formação está em um problema de colonialidade do ser e do saber, porque o currículo continua privilegiando o conhecimento técnico sobre a formação humana. A tecer a bioética intercultural da reflexão pedagógica em contextos interculturais permite desenvolver um treinamento crítico sobre o que não favorece a harmonia do ser humano com o território e que leva o futuro profissional a ter consciência de sua responsabilidade com os cuidados em saúde.

Palavras chave: Enfermagem, cuidado, interculturalidade, bioética, educação.

\section{INTRODUCCIÓN}

La educación requiere de procesos que propendan por mejorar las capacidades de las personas, procurando su bienestar y dignidad. Es decir, adoptar una visión humanista de la educación que se adapte a las necesidades de las culturas, teniendo en cuenta su pluralidad y aceptando tanto otros modelos educativos como otros sistemas de conocimiento (Unesco, 2015). En Colombia, este acercamiento se ha intentado lograr por medio de la legislación en materia de 
Revista científica de la Asociación de Historia y Antropología de los Cuidados (Universidad de Alicante)

educación y salud, pero aún hay camino por recorrer, fundamentalmente en las universidades, las cuales deben ser las principales involucradas con este propósito en razón a que la responsabilidad de la humanización de la educación recae en ellas.

Considerando que, la educación requiere procesos de de-colonización de las pedagogías y de los contenidos curriculares, respondiendo a una visión de aprendizaje que sea organizado, pero en los casos que sean necesarios se adapte y se transforme según los requerimientos del contexto (Unesco, 2015). En este sentido, el área de la salud debe cambiar su perspectiva de formación, que se centra en un enfoque biologicista, el cual resulta reduccionista al tener en cuenta que "la salud es un estado de completo bienestar físico, mental y social, y no solamente la ausencia de afecciones o enfermedades" (Organización Mundial de la Salud, 1948), por una visión que reconozca al ser humano como responsable del cuidado de su salud, la cual depende no solamente de su fisiología, sino que es producto de su interrelación con el contexto y la visión cultural propia de salud.

De igual modo, la enfermería requiere de la humanización para tener coherencia con su propósito, el cuidado, que desde la visión antropológica de Leininger (1999) se define como las acciones encaminadas a la mejora de las condiciones o estilo de vida de una persona que se encuentra en necesidad o se prevé la misma, lo anterior a través de la asistencia, apoyo y facilitación de medidas para el bienestar del sujeto de cuidado, lo cual debe ser visto desde una perspectiva holística (Moreno y Muñoz, 2016). Así, desligarse del lado humano es alejarse del objeto de la disciplina, pasando a ser solo la aplicación de técnicas. Es lo que Fawcett (2005) considera una visión de reacción, que lo reduce a lo biológico e ignora otras variables que influyen en la salud del sujeto considerándolo homogéneo. Es por esto que humanizar la enfermería en su formación equivale a humanizar el cuidado; es llegar a una visión de reciprocidad, requiriendo una formación bioética que aporte las condiciones para analizarlo. Este postulado se acerca más a la mirada latinoamericana, que lo asume como una reflexión sobre perspectivas éticas y morales con respecto a la vida para tomar decisiones, donde la cultura es determinante para el entendimiento. Lo anterior en razón a que todo asunto personal es un asunto social a la vez, considerando una visión contextualizada del problema que le da importancia a la voz del sujeto de cuidado y lo vuelve protagonista (Álvarez-Díaz, 2012).

Esta necesidad de formar en bioética a los profesionales de enfermería se hace urgente en contextos diversos como el colombiano, para responder a las necesidades propias de un cuidado con enfoques epistémicos y bioéticos novedosos que posibiliten la reflexión en torno a los problemas que derivan de la salud por la persona y el contexto, incluyendo un 
enfoque intercultural. Para esto se requiere de formación en actitudes y aptitudes que posibiliten el trato y la comunicación; pero esto no es simplemente incluirlo en el currículo, sino que se necesita lograr una internalización del concepto de la bioética y adoptar una perspectiva intercultural que busque por medio de las relaciones con los otros, en la praxis, conocerse y reconocerse como alguien distinto para conocer $\mathrm{y}$ reconocer al otro, lo que posibilita una comunicación efectiva que incluye las diferencias (Bartolomé, 2006; Fornet, 2009).

De ahí que humanizar el currículo equivale a incluir perspectivas interculturales; es decir, que la universidad cuente con apertura epistémica, ontológica y política en cuanto a distintas perspectivas en torno a la salud, contribuyendo a una formación bioética desde un currículo pertinente, que no se construye para el contexto, sino desde el mismo (Unesco, 2015). Esta pertinencia curricular hace referencia a la defensa de la libertad frente a la estandarización, al respeto e inclusión de la diferencia sobre la homogeneización; es abogar por la misión y el espíritu universitario (De Zubiría, 2016). Lo anterior debe verse reflejado en un currículo que corresponda a la construcción de un orden específico de un discurso, según la situación histórica y social en la que está inmerso (Kemmis, 2008), para así formar en la reflexión, preparando a los estudiantes en la convivencia con otros, donde la práctica posiciona al estudiante como autónomo y le exige la reflexión crítica, a la vez que se le permite asumir las decisiones y las consecuencias de estas (Vasco Uribe, 1990). Ahora bien, para acercarse a los resultados del proceso de formación, teniendo en cuenta que el propósito de este es lograr que se interioricen conceptos y se establezcan modos de comportarse según lo que se ha enseñado, se hace relevante hablar del sentido, entendido como una construcción que se genera a partir de la percepción que se da a través de los sentidos y que se nutre de las relaciones del yo con el otro y el contexto (Holzapfel, 2006). De esta manera, en este artículo se busca comprender los sentidos de la bioética que emergen de la formación de enfermeros en contextos interculturales, teniendo en cuenta que en el contexto colombiano, y específicamente el Cauca (departamento de Colombia), la diversidad cultural y étnica (población indígena del 20.5\%; afro descendiente del $21.1 \%$ y mestiza-blanca del 58.5\% (Departamento Administrativo Nacional de Planeación, 2014)) convierte en un reto la toma de decisiones para los profesionales de enfermería, en razón al choque que se produce constantemente entre la cosmovisión de salud de los sujetos de cuidado y la formación que recibieron, donde impera un saber técnico que no permite darle voz a dichos sujetos.

\section{METODOLOGÍA}

La investigación realizada es de carácter cualitativo de tipo descriptivo, puesto que buscaba conocer fenómenos 
Revista científica de la Asociación de Historia y Antropología de los Cuidados (Universidad de Alicante)

desde la perspectiva del interlocutor, para así describir el objeto de estudio (Arráez, Calles y Moreno, 2006; Sánchez y Sánchez, 2010). De enfoque hermenéutico, pretendiendo ir más allá de lo biológico y técnico, permitiendo visibilizar de manera precisa y detallada los contenidos de los discursos de los participantes para identificar los sentidos que perciben desde su realidad. El análisis se hizo desde la teoría fundamentada (Do Prado, De Souza y Carraro, 2008), que se usó para conocer los detalles del fenómeno estudiado y a partir de ellos establecer nuevos conocimientos, los cuales emergen de las relaciones, permitiendo establecer teorías.

Esta investigación se realizó en el Programa de Enfermería de la Universidad del Cauca, en la ciudad de Popayán (Colombia), y en el hospital Mama Dominga, ubicado en el Resguardo Indígena de Guambía (municipio de Silvia, departamento del Cauca, Colombia), en el marco de la práctica formativa comunitaria de los estudiantes de último semestre. Los participantes, denominados actores de formación, se seleccionaron según criterios de inclusión y exclusión. Así, se contó con la colaboración de 8 estudiantes, 14 profesionales entre directivos del programa (decano, jefe de Departamento, coordinadora del Programa y miembros del Comité Académico) y docentes (de planta, ocasionales de tiempo completo y jubilados) que han formado en bioética a los estudiantes seleccionados y han participado en los procesos de acreditación del programa. También participaron 6 profesionales que ejercen como tutores de práctica (personas que están vinculadas a la institución de salud antes mencionada, encargadas de realizar seguimientos $y$ acompañar a los estudiantes), y 5 sujetos de cuidado (pertenecientes al Resguardo Indígena Misak) que fueron atendidos por los estudiantes de práctica. Adicionalmente, se revisaron 33 documentos de orden nacional y local referentes a educación en salud.

Los datos fueron obtenidos entre el 2017 y el 2018, utilizando técnicas cualitativas para la recolección de información tales como: revisión documental de 33 documentos, 9 entrevistas semiestructuradas, observación no participante registrada en 17 diarios de campo y 5 grupos focales. Se empleó el criterio de saturación teórica y la revisión por pares externos expertos, con el fin de no perder el horizonte de estudio, apoyando el proceso de interpretación.

El análisis de datos se realizó en tres fases siguiendo la teoría fundamentada, teniendo en cuenta que el objetivo era conocer los sentidos que emergen en la formación a través de los discursos, generando esquemas explicativos. La primera fase consistió en la recolección de datos. En la segunda fase se efectuaron las codificaciones abierta y axial: la primera se refiere al microanálisis línea por línea en donde se descomponen los datos para 
categorizarlos, por medio del sublineado colorido que permitió separar temas e ideas centrales que definieron categorías, y en la segunda se trabajó en las relaciones entre las categorías y subcategorías que se encontraron en la codificación abierta (Strauss y Corbin, 2002), de las cuales surgieron las variables centrales. Por último, en la tercera fase se hizo la codificación selectiva, donde se vislumbraron los núcleos temáticos, generando una teoría de la integración y refinamiento de lo encontrado para lo cual se organizaron las variables y categorías en torno a un concepto explicativo central, a partir de las concepciones sobre el fenómeno estudiado (Strauss y Corbin, 2002). Así, la teorización corresponde a la explicación principal que se aportó con base a la condensación de las relaciones de las categorías y subcategorías, como también de las variables centrales, generando esquemas explicativos.

En cuanto a los aspectos éticos, este estudio fue avalado por el Comité Ético de la Vicerrectoría de Investigaciones de la Universidad del Cauca, contando previamente con la autorización tanto del Programa de Enfermería como del Hospital Mama Dominga. Se resguardó la privacidad de los participantes y se contó con el consentimiento informado.

\section{RESULTADOS}

Los hallazgos sobre los sentidos fueron analizados según cuatro categorías, las cuales se describieron de acuerdo con los documentos (sentido semántico) y los actores de formación (sentido existencial). Los sinsentidos encontrados en los discursos se plantearon como el trasfondo de sentido y corresponden a un extracto de la realidad, señalando lo distanciada que está la experiencia formativa de los objetivos establecidos en papel. A partir de estas, se establecieron las cuatro variables centrales a discutir en el análisis (Tabla 1).

\section{DISCUSIÓN Y CONCLUSIONES}

Teniendo en cuenta los resultados obtenidos, a continuación se presenta el análisis de las variables centrales que representan las voces de los actores y los sentidos conferidos a la formación bioética.

\section{Pertinencia curricular, una} tarea por cumplir.

Es definida desde los sentidos y sinsentidos como una formación bioética que supla las necesidades relacionadas al contexto cultural diverso. No obstante, la formación se ha centrado en la descripción de técnicas y aptitudes que tienden a homogeneizar el ser y el hacer, situación que imposibilita una visión más humana del cuidado. Al respecto, un profesor que participó en la investigación comentó:

"[Los estudiantes dicen:] ¿Para qué me meto en ética? A mí lo que me interesa es pasar una sonda nasogástrica y pasarla bien, y hacerlo correctamente desde lo 
Revista científica de la Asociación de Historia y Antropología de los Cuidados (Universidad de Alicante)

técnico, y explicarle al paciente el procedimiento. ¿Lo estoy teniendo en cuenta a él como ser humano?

¡No! (Docente de Enfermería, entrevista personal, 18 de junio de 2018. Cursivas añadidas) ${ }^{1}$

Además, se espera que lo normativo (tanto en la universidad como en las instituciones prestadoras de servicios de salud) sea la guía para el diálogo universidad y contexto, pero en ocasiones se convierte en un obstáculo para quien forma, al limitar su rol al cumplimiento del conocimiento oficial desde lo que es válido científicamente. Aquello no da cabida al reconocimiento de otros saberes que emergen desde las prácticas (Becerra, 2014). De ahí que la práctica formativa se vislumbra como clave para superar este problema en la formación bioética, al dar paso al encuentro entre el yo y la otredad, permitiendo reconfigurar el currículo desde una perspectiva flexible que se adapte al contexto, superando así la mirada biologicista de fundamentación técnica que prima en la formación (De Zubiría, 2016).

Un punto de partida para decolonizar el ser y el saber (Mignolo, 2007) en la construcción del currículo, es en el acto pedagógico, donde el profesor como modelo de formación bioética, desde su praxis, genera "condiciones en las que todas las personas pueden lograr un bienestar máximo

\footnotetext{
${ }^{1}$ Por petición de las personas participantes en la investigación, sus nombres no son revelados en este artículo, preservando así
}

$\mathrm{y}$ un total potencial integrado con el conocimiento ético, estético, personal, empírico y con el saber en la práctica de enfermería" (Chin y Kramer, 2008, p. 302). Esto aporta sentido en la medida en que, desde el acto pedagógico, el docente como maestro muestra actitudes de respeto, humildad y escucha a los estudiantes para construir con ellos su dimensión moral (Zaror, Muñoz, Espinoza, Vergara y Valdés, 2014), situación que se ve reflejada en la relación que establece el estudiante con las personas en el acto de cuidado: "Hay docentes que te enseñan a ser persona, hay otros que solo se limitan a enseñarte la teoría, y la teoría no es siempre lo que uno necesita en estos casos" (Estudiante de Enfermería 1, intervención en grupo focal, 24 de octubre de 2017).

Se plantea, entonces, la construcción de un currículo que escuche al contexto al formar de acuerdo a sus necesidades y saberes. Luego se espera que haya una articulación entre elementos cognoscitivos y actitudinales, porque no solo basta con mostrar, entender $\mathrm{o}$ reconocer un contenido bioético intercultural para homogeneizar, sino que se requiere cambiar el papel que se le asigna a los contenidos en los planes y programas de estudio, donde la pedagogía se abre como espacio de reflexión: Tiene que ser desde nuestro contexto, con las

la confidencialidad concertada desde un comienzo. 
Cultura de los Cuidados

condiciones de nuestro

contexto y no lo que nos

están diciendo desde

Europa, Norteamérica [...]

Los problemas de allá no

nos sirven acá y desde la

práctica misma, cuando yo

estoy en una práctica y

encuentro que la paciente ni

siquiera tiene plata [dinero]

para comprar una jeringa,

que no tienen dinero para

pagar el bus que los traiga al

hospital en un momento de

crisis, ¿cómo voy hablar yo

de que el problema que

tengo que trabajar es la

fertilización in vitro?

(Docente de Enfermería, entrevista personal, $12 \mathrm{de}$ junio de 2018. Cursivas añadidas)

\section{Aprender a convivir para} comprender el cuidado de la salud como territorio.

Entre los actores participantes se percibe la bioética como un concepto inacabado que se reconfigura a partir de las experiencias de aprendizaje en los contextos de práctica, en la interacción con los otros en el acto de cuidado, donde emergen relaciones y choques derivados de los cuestionamientos entre su formación y las diferentes visiones sobre el mismo. Aquello lleva al reencuentro del profesional con la razón de ser de la disciplina: el cuidado.

Sobre este aspecto, un alumno indicó:

$$
\begin{aligned}
& \text { Se hace necesario conocer la } \\
& \text { cultura para tratar de mediar } \\
& \text { con situaciones en las cuales } \\
& \text { pueden existir choques por } \\
& \text { la manera en cómo cada una } \\
& \text { concibe las diferentes } \\
& \text { formas de vivir [...] Nunca } \\
& \text { se debe pasar a juzgar los } \\
& \text { comportamientos sin } \\
& \text { conocer los significados [en } \\
& \text { salud] que tienen para las } \\
& \text { diferentes culturas. } \\
& \text { (Estudiante de Enfermería } \\
& 7 \text {, intervención en grupo } \\
& \text { focal, } 24 \text { de octubre de } \\
& 2017 \text { ) }
\end{aligned}
$$

Es decir, los estudiantes requieren formarse en diversas miradas del ser y el conocer en torno al cuidado, para abandonar los prejuicios durante el proceso de formación, al contar con teorías contextualizadas desde la lógica local y el territorio. Por tanto, el cuidado en un contexto diverso es un reto que implica considerar y respetar al sujeto de cuidado, incluyendo sus creencias, valores $\mathrm{y}$ costumbres (Ibarra y Siles, 2006). Así, el tener en cuenta la cosmovisión de dichos sujetos permite comprender que el cuidado de la salud en estos contextos supera la visión biologicista hegemónica de la enfermedad, porque para ellos la salud es territorio. Por consiguiente, se cuida de un 
Revista científica de la Asociación de Historia y Antropología de los Cuidados (Universidad de Alicante)

territorio en donde la persona vive y convive en armonía con todo lo que le rodea, priorizando el papel de la comunidad y el efecto en la salud de sus miembros. Esta situación genera un giro en torno a la visión de cuidado, lo que proporciona fundamento moral a la práctica de la profesión de enfermería, y permite, en la bioética, el análisis de las condiciones para hacer posible el cuidado en estos contextos.

De ahí que los actores demanden sobre la bioética, en la práctica, un proceso de formación del futuro profesional para aprender a convivir, pensando en comunidad $\mathrm{y}$ en contexto, que no solo es lo humano desde la perspectiva del vivir bien (Huanacuni, 2010). Aquello implica, en el acto de cuidado, desaprender para volver a aprender, al cuestionar al estudiante desde lo vital y permitiéndole dar paso a reconocer al otro en su horizonte (Fornet, 2001). Al mismo tiempo, se permite ser críticos respecto a aquello que no favorece la vida o que niega la dignidad humana (Fornet, 2007), por lo cual se requiere de estrategias de autoconocimiento que lleven a reflexionar sobre el aprendizaje y las formas de aprender desde la práctica (Ayala, Alvarado y Cuevas, 2010).

\section{Relaciones donde la barrera no} es el lenguaje, es la actitud.

Los hallazgos alcanzados en la investigación señalan que, en la formación bioética, tanto el acto pedagógico como el acto de cuidado se construyen en las relaciones que emergen en el contexto de práctica diverso culturalmente, ya sea entre el docente y el estudiante o entre el cuidador y el sujeto de cuidado. Aquí el lenguaje no es la barrera, lo es la actitud bajo la cual se ha formado al futuro profesional. Esto en razón a que uno de los principales obstáculos es la comunicación, al carecer de una actitud de escucha que propenda por comprender al otro. Se reclama de la interculturalidad, entonces, que sea un puente en la transformación de la formación bioética, porque no solo basta con reconocer al otro en su diferencia o aprender un lenguaje para relacionarse con él, sino formar, desde relaciones de apertura a otras miradas del cuidado de la salud, en búsqueda de lo que nos hace iguales. Se trata de una "interculturalidad centrada desde la mirada de uno mismo, desde donde uno se sitúa, desde donde uno mira al otro" (Docente de Enfermería 7, entrevista personal, 12 de junio de 2018).

Un punto de partida para ello es la articulación de las universidades y los campos de práctica, al considerar las necesidades del contexto y aportar a la comunidad no solo desde el conocimiento técnico, sino desde la interacción humana afectiva, efectiva y respetuosa en el tiempo. De esta forma, las experiencias de aprendizaje del estudiante y del docente, así como las necesidades del contexto que trascienden del tiempo inmediato del acto de cuidado, forjan la de-construcción de una temporalidad como condición de 
permanente renovación, posibilitando la reconstrucción del currículo. Para esto se proponen espacios de socialización cultural en prácticas comunitarias tempranas $\mathrm{y}$ concertadas en contextos diversos, donde el primero que debe realizar estas inmersiones es el profesor, con el fin de posibilitar su rol de formador bioético con una visión de cuidado más humana. El docente debe además formar con una perspectiva del cuidado de la salud que supere la mirada individual propia de las culturas occidentales, al tener en cuenta que el aprendizaje intercultural se da a través de los choques, la reflexión de lo enseñado en el aula y la realidad en el contexto de práctica, lo que abre paso a una formación bioética.

Por tanto, se requiere "romper la 'fatal' pretensión del científico por la verdad objetiva y hacer del conocimiento una práctica comprometida que problematiza los escenarios de acción, pero sin acción concreta su efecto es realmente fútil" (Díaz, 2012, p. 41). Así mismo, es necesario reconocer el cuidado como una responsabilidad compartida que, al igual que en la visón intercultural, se asume desde la perspectiva de vivir en comunidad, de tener reciprocidad, donde todos aportan y todos reciben (CRIC, s.f.). Tal y como lo mencionó un alumno:

$$
\begin{aligned}
& \text { Compartir nuestros } \\
& \text { conocimientos, pero de una } \\
& \text { manera [humilde], no que } \\
& \text { sabemos todo, sino de una } \\
& \text { manera que ellos }
\end{aligned}
$$

[refiriéndose a los sujetos de cuidado] nos enseñan cosas a nosotros y nosotros les enseñamos cosas a ellos. Si ellos lo toman, pues les empezamos a explicar más; si no lo toman, lo dejamos ahí. (Estudiante de Enfermería 1, diario de campo, 29 de septiembre de 2017)

\section{Cuidado del nosotros, pensando} en el otro como uno mismo.

Se asume un cuidado del nosotros al reconocer al otro como un igual, que es responsable de su cuidado, lo que implica reconfigurar lo pedagógico, lo curricular y lo disciplinar hacia una visión más humana, para de-construir la barrera de formación bioética que está en nosotros mismos. Lo anterior con el fin de que el futuro profesional se sienta parte del contexto y no como un extraño, permitiendo una formación bioética con visión crítica que, en la práctica y en las relaciones que emergen en el acto de cuidado, le concedan abstraer sentidos y le ayuden a construir una visión más humana.

Se exige, además, una pedagogía del conocer y del vivir donde el docente enseñe desde su experiencia, como ejemplo, y considere a sus estudiantes como iguales en importancia, requiriendo que se forme a quienes forman para que ellos cuenten con un referente epistémico y ontológico. Dado 
Revista científica de la Asociación de Historia y Antropología de los Cuidados (Universidad de Alicante)

que la bioética permite la reflexión sobre las situaciones donde se realiza el cuidado y la interculturalidad se establece como un puente para acercar al futuro profesional y al sujeto de cuidado: "Si se desea que el estudiante sea una persona que cuide, el proceso de enseñanza-aprendizaje se plasma de acciones de cuidado que visualicen el lado humano de la educación" (Guerrero y Costa, 2019, p. 2), reiterando que el primer camino es la reflexión pedagógica que reconstruya el currículo.

En consecuencia, el respeto del estudiante hacia el sujeto de cuidado emerge de la relación docente-estudiante que, como modelo de formación, le muestra la forma de asumir las relaciones en el acto mismo del cuidado (Noddings, 2005). En este sentido, la formación bioética desde la práctica contribuye a de-colonizar la visión ontológica y epistémica en la cual ha sido formado el estudiante, debido a que en ella puede utilizar sus conocimientos y generar aprendizajes significativos (Sánchez y Rondón, 2013). Este espacio confiere sentidos al estudiante al ser reconocido en la comunidad como profesional que merece respeto $\mathrm{y}$, en consecuencia, le brinda autonomía al fortalecer su identidad profesional para que asuma una postura responsable con las personas en el acto de cuidado, al sentirse parte del nosotros: “Te pones en la posición de la otra persona $\mathrm{y}$ cuidas desde la percepción de la otra persona, [desde lo que necesita], no imponiendo lo que sabes" (Estudiante de
Enfermería 1, diario de campo, 29 de septiembre de 2017).

Tejer la bioética intercultural como categoría central: a modo de conclusión.

De las anteriores variables emergió la categoría central tejer la bioética intercultural que, desde la reflexión pedagógica en los contextos interculturales de la práctica profesional de enfermería, favorece la de-construcción del currículo en la formación bioética hacia una visión más humana del cuidado. Por consiguiente, las experiencias de aprendizaje de la bioética como un campo que conjuga conocimientos $\mathrm{y}$ actitudes, se pueden internalizar desde el contacto con el otro en los campos de práctica. Lo anterior en razón al choque con otras visiones de salud en el acto de cuidado $\mathrm{y}$ en el acto pedagógico, y desde el reencuentro consigo mismo (de quien forma y a quien se forma) para asumir una postura frente al quehacer profesional y su responsabilidad compartida con el sujeto de cuidado en torno a la salud. Así, la bioética intercultural actúa como un puente que decoloniza el ser y el conocer, porque suscita en los futuros profesionales de enfermería una nueva actitud que cambia la forma en cómo ven y se acercan a las personas.

Un primer punto para tejer la bioética intercultural implica un cuidado de nosotros, lo que supone una responsabilidad profesional que se asume como colectiva, a partir de la reflexión crítica sobre la visión moral del quehacer profesional, teniendo en cuenta la cultura y el contexto de las 
personas. Aquello se origina en el

fortalecimiento de la identidad profesional, que empieza en las relaciones que establecen los estudiantes con el docente en el acto pedagógico, quienes se convierten en los primeros modelos de formación del cuidado, porque si el estudiante no observa al profesor realizar acciones éticas con fundamento epistémico y ontológico, su visión de cuidado quedará reducida a una técnica que lo aleja de la relación humana. Por otra parte, al sentirse reconocido como profesional por parte de los sujetos de cuidado y del equipo de trabajo, el estudiante adquiere autonomía en la toma de decisiones y en la capacidad afectiva.

De ahí que quien cuida reconoce a quien es cuidado como alguien que no soy yo, pero somos a la vez, donde no hay lugar solo para tolerar otras visiones del cuidado, porque supondría superioridad en el sentido en que prima un saber $y$ un hacer hegemónico, sino que se propende por una relación dialógica del reconocimiento del otro en los puntos de encuentro en torno a la salud. Esto conlleva a sentirse parte de un nosotros, en un contexto que le permite al cuidador reconocerse y ser reconocido para así pasar a la de-colonialidad del ser, abandonando la posición hegemónica $\mathrm{y}$ técnica en que fue formado.

Forjada la identidad profesional, un segundo punto se teje a partir de relaciones donde el lenguaje no es la barrera, porque no solo basta con reconocer la diversidad, sino que es necesaria la acción hacia la práctica activa de la escucha y el contacto tanto en el acto pedagógico como en el acto de cuidado. La escucha en un sentido existencial que trasciende lo biológico, debido a que hacemos parte de un territorio donde somos o podemos ser. Se trata de una actitud de apertura al ser y conocer hacia las perspectivas del otro en torno al cuidado de la salud, dando paso al contacto como una vivencia desde un encuentro no solo físico sino epistémico y ontológico, donde se aprende no para el otro sino desde el otro. De esta manera, se plantea una formación bioética que invite a reconstruir un currículo desde la relación entre la universidad y el contexto, en búsqueda de objetivos comunes de formación que aporten al territorio, construyan permanentemente el currículo y resignifiquen la posición normativa bajo la cual se ha mantenido colonizado el conocer.

En efecto, tejer la bioética intercultural es un ciclo formativo $\mathrm{y}$ transformativo que incluye el cuarteto sujeto que forma, sujeto al que se forma, sujeto de cuidado y contexto, donde los tres aprenden, enseñan y sanan en un acto de convivencia que tiene sentido vital, es decir, que da vida al de-colonizar el ser y el conocer, dando paso a un tercer punto: aprender a convivir. Precisamente, el convivir es concebir que el futuro profesional hace parte de una comunidad, cuyas visiones en torno al cuidado de la salud contribuyen a reconstruir su visión de cuidado, donando el fundamento moral del futuro profesional.

En consecuencia, se amplía el campo de reflexión en torno a la bioética desde las particularidades del contexto 
Revista científica de la Asociación de Historia y Antropología de los Cuidados (Universidad de Alicante)

permeadas por una cultura, entendiendo que la bioética es un concepto inacabado que se reconstruye en las relaciones con los otros. Las prácticas en contextos interculturales permiten al estudiante reconocerse como un ser humano que hace parte de un territorio, el cual es salud y, por tanto, es llamado a cuidar ese territorio, a cuidarse a sí mismo. Aquello se presenta cuando se incluyen en los procesos de formación bioética estrategias de autoconocimiento que le permiten al futuro profesional de enfermería transitar del aprendizaje a la comprensión, al considerarse como un agente de cambio que debe construir su propia visión de cuidado más humana, fortaleciendo su identidad profesional y asumiendo su responsabilidad como tal desde un nosotros.

Como resultado de todo lo anterior, de la de-colonidad del ser y el conocer en la formación bioética intercultural, se consolida la pertinencia curricular, al considerar la praxis y la pedagogía como procesos de reflexión permanente, frente a la conducta y responsabilidad de los profesionales de enfermería con las personas y su territorio en el acto de cuidado. La praxis, desde el maestro, propende por desarrollar en el estudiante modos de razonamiento práctico en el acto pedagógico, al concederle criterios bioéticos para decidir modos de acción cuando se enfrenta a los choques de orden moral en el acto de cuidado. De manera que la pedagogía del vivir y el conocer considera el vivir desde un docente como maestro, quien desde su ejemplo de formación no entra en contradicciones, y el conocer lo dota de un sustento epistémico y ontológico de la visión disciplinar del cuidado, con la cual orienta a los estudiantes. Ello requiere que el mismo docente se forme y pueda superar la tendencia profesionalizante, continuando hacia la tendencia disciplinar desde un enfoque investigativo, siguiendo un camino por medio del cual el currículo se construye permanentemente. Es ahí donde el maestro, consciente de que el estudiante es un actor protagónico de su formación, reconoce su importancia como donador de sentido al proceso formativo bioético, y abre la posibilidad de un tránsito efectivo de una visión de mundo que va de lo técnico a la humanización de la educación.

Agradecimientos: Agradezco a la Universidad del Cauca, al Departamento de enfermería y al Hospital Mama Dominga por el apoyo brindado para la realización del presente trabajo.

No se contó con financiamiento para esta investigación y no se presentaron conflictos de intereses.

\section{BIBLIOGRAFÍA}

Álvarez-Díaz, J. (2012). ¿Bioética latinoamericana o bioética en Latinoamérica? Revista Latinoamericana de Bioética, 12(22), 11-27. Doi: https://doi.org/10.18359/rlbi.989

Arráez, J., Calles, J., y Moreno, L. (2006). La hermenéutica: una actividad interpretativa. Revista Universitaria de Investigación, 7(2), 171-181. Recuperado de https://www.redalyc.org/pdf/410/41070212. pdf 
Ayala, R., Alvarado, C., y Cuevas, J. (2010).

La discriminación en el aprendizaje del cuidado. Ciencia y Enfermería, 16(3), 85101. Doi:https://dx.doi.org/10.4067/S07179553201000030001

Bartolomé, M. A. (2006). Procesos interculturales. Antropología política del pluralismo cultural en América Latina. México: Siglo XXI.

Becerra, A. (2014). El cuidado en enfermería: Una propuesta de formación a partir de la experiencia en la ciudad de Cali (Tesis de Doctorado). Facultad de Ciencias Naturales, Exactas y de la Educación, Universidad del Cauca: Popayán, Colombia.

Chin, P., y Kramer, M. (2008). Integrated theory and knowledge development in nursing. St Louis: Elsevier Mosby.

CRIC -Consejo Regional Indígena del Cauca-. (s.f.). Programa de salud. Sitio web del CRIC Colombia. Recuperado de https://www.cric-

colombia.org/portal/proyecto-

cultural/programa-de-salud

De Zubiría, S. (2016). Sentido social de la universidad: dilemas y potencialidades. En D. M. Rodríguez (Ed.), Bioética. Ecología de saberes. ¿La vida debe tener prioridades sobre los intereses de la ciencia? (pp. 3749). Bogotá: Universidad Libre.

Departamento Administrativo Nacional de Planeación. (2014). Censo Nacional Agropecuario. Bogotá: DANE.

Díaz, E. (2012). Una mirada a las contradicciones de la revitalización lingüística en el Cauca. Revista Tabula Rasa, 1(17), 219-244. Recuperado de http://www.scielo.org.co/pdf/tara/n17/n17a 11.pdf

Do Prado, M., De Souza, M., y Carraro, T. (2008). Investigación cualitativa en enfermería: contexto y bases conceptuales. Brasil: Organización Panamericana de la Salud.
Fawcett, J. (2005). Criteria for Evaluation of Theory. Nursing Science Quarterly, 18(2), 131-135.

Doi: https://doi.org/10.1177/0894318405274823

Fornet, R. (2001). Transformación intercultural de la filosofía. Bilbao: Desclée de Brouwer.

Fornet, R. (2007). Reflexiones de Raúl Fornet-Betancourt sobre el concepto de Interculturalidad. México: Consorcio Intercultural.

Fornet, R. (2009). Interculturalidad en procesos de subjetivización. México: Consorcio Intercultural.

Guerrero, R. F., y Costa, J. L. (2019). El estudiante de enfermería como ser-cuidado en la relación docente-estudiante: reflexión en Heidegger. Presencia, Revista Internacional de Salud Mental, Investigación y Humanidades, 5(s.n.), 1-5. Recuperado de http://ciberindex.com/c/p/e12290

Holzapfel, C. (2006). A la búsqueda del sentido. Revista de Filosofía, 62(s.n.), 163166. Doi: http://dx.doi.org/10.4067/S071843602006000100011

Huanacuni, F. (2010). Buen Vivir/Vivir Bien. Filosofía, politicas, estrategias $y$ experiencias regionales andinas. Lima, Perú: Coordinadora Andina de Organizaciones Indígenas.

Ibarra, T., y Siles, J. (2006). Competencia cultural. Una forma humanizada de ofrecer cuidados de enfermería. Índex de Enfermería, 15(55), 44-48. Recuperado de http://scielo.isciii.es/scielo.php?script=sci_a rttext\&pid $=$ S1132$12962006000300010 \& \operatorname{lng}=\mathrm{es}$

Kemmis, S. (2008). El currículum: más allá de la teoría de la reproducción. Madrid, España: Morata.

Leininger, M. (1999). Cuidar a los que son de culturas diferentes requiere el conocimiento y las aptitudes de la 
enfermería transcultural. Cultura de los cuidados, 5-12.

Mignolo, W. (2007). El pensamiento decolonial: desprendimiento y apertura. En S. Castro y R. Grosfoguel (Eds.), El giro decolonial. Reflexiones para una diversidad epistémica más allá del capitalismo global (pp. 25-46). Bogotá: Siglo del Hombre Editores.

Moreno, M., y Muñoz, L. (2016). De la teoría de enfermería a la práctica: experiencias con proyectos de gestión del cuidado. Chía, Colombia: Universidad de la Sabana.

Noddings, N. (2005). The challenge to care in schools. An alternative approach to education. Nueva York, Estados Unidos: Teacher College Press.

Organización Mundial de la Salud. (1948). Conferencia Sanitaria Internacional. Documento registrado en Official Records of the World Health Organization, Nueva York, Estados Unidos.

Sánchez, G., y Sánchez, R. (2010). Hermenéutica ¿nueva koiné?: ¿Cómo reducir su relativización? En J. M. Cuartas (Comp.), Hermenéutica en Acción (pp. 4150). Cali, Colombia: Editorial Universidad del Valle.
Sánchez, M., y Rondón, B. (2013). La diversidad cultural en los procesos de formación académica de enfermería requiere el manejo de la ética pedagógica, la corresponsabilidad y un pensamiento mediador. Enfermería Global, 12(29), 147$157 . \quad$ Recuperado de http://scielo.isciii.es/pdf/eg/v12n29/docenci a2.pdf

Strauss, A., y Corbin, J. (2002). Bases de la investigación cualitativa: técnicas y procedimientos para desarrollar la teoría fundamentada. Medellín: Universidad de Antioquia.

Unesco -Organización de las Naciones Unidas para la Educación, la Ciencia y la Cultura- (2015). Replantear la educación: ¿Hacía un bien común mundial? París, Francia: Ediciones Unesco.

Vasco Uribe, C. (1990). Algunas reflexiones sobre la pedagogía y la didáctica. Bogotá: Editorial El Griot.

Zaror, C., Muñoz, P., Espinoza, G., Vergara, C., y Valdés, P. (2014). Enseñanza de la bioética en el currículo de las carreras de odontología desde la perspectiva de los estudiantes. Acta Bioética, 20(1), 135-142. Doi: $\quad$ https://dx.doi.org/10.4067/S1726$\underline{569 \times 2014000100015}$

Tabla 1

Sentidos semántico, existencial y trasfondo de sentido

\begin{tabular}{|c|c|c|c|c|}
\hline Categoría & $\begin{array}{c}\text { Sentido } \\
\text { semántico }\end{array}$ & Sentido existencial & $\begin{array}{c}\text { Trasfondo de } \\
\text { sentido }\end{array}$ & $\begin{array}{c}\text { Variable } \\
\text { central }\end{array}$ \\
\hline $\begin{array}{l}\text { Prácticas formativas en } \\
\text { la bioética. }\end{array}$ & $\begin{array}{l}\text { Con base a los } \\
\text { objetivos } \\
\text { institucionales y } \\
\text { necesidades del } \\
\text { contexto (pertinencia), } \\
\text { se busca la formación } \\
\text { para el desarrollo } \\
\text { integral de los } \\
\text { estudiantes en pro de } \\
\text { la autonomía, la toma } \\
\text { de decisiones, el } \\
\text { pensamiento crítico, la } \\
\text { ética y la inteligencia } \\
\text { emocional y social, } \\
\text { siendo la práctica un } \\
\text { escenario de } \\
\text { interacción y }\end{array}$ & $\begin{array}{l}\text { En el currículo se reduce a } \\
\text { contenidos privilegiando lo } \\
\text { conceptual y técnico, } \\
\text { quedando relegada la } \\
\text { formación del ser. La } \\
\text { formación que se produce } \\
\text { a través del aprendizaje } \\
\text { mediante el acercamiento } \\
\text { con el otro en el territorio, } \\
\text { se ve obstaculizada en } \\
\text { razón a que las prácticas se } \\
\text { centran en el desarrollo de } \\
\text { habilidades técnicas y estas } \\
\text { son señaladas como } \\
\text { insuficientes para dar } \\
\text { respuesta a un contexto } \\
\text { diverso, que demanda de }\end{array}$ & $\begin{array}{l}\text { Tendencia de formación } \\
\text { biologicista fundamentada en } \\
\text { la técnica que imposibilita } \\
\text { asumir una visión más } \\
\text { humana del cuidado que } \\
\text { incluya la cosmovisión y el } \\
\text { territorio, en razón a la } \\
\text { homogeneidad del ser y el } \\
\text { hacer, que enfatiza aptitudes y } \\
\text { no actitudes que le permitan } \\
\text { asumir la reflexión y crítica } \\
\text { frente a la responsabilidad } \\
\text { social de la profesión y el } \\
\text { contexto: } \\
\text { "Uno observa un valor de } 10 \\
\text { \% en la evaluación del ser } \\
\text { [...] La evaluación es un }\end{array}$ & $\begin{array}{l}\text { Pertinencia } \\
\text { curricular, una } \\
\text { tarea por cumplir. }\end{array}$ \\
\hline
\end{tabular}


Cultura de los Cuidados

transformación del

hacer clínico

fundamentado en una

visión humanista. " $E l$

propósito de fortalecer

y generar

competencias,

capacidades y nuevos

conocimientos en los

estudiantes y docentes

de los programas de

formación en salud, en

un marco que

promueve la calidad

de la atención y el

ejercicio profesional

autónomo,

responsable y ético de

la profesión"

sobre la relación

docencia-servicio en

salud de 2010).
(Documento nacional

los estudiantes una actitud

de disposición y apertura

que permita escuchar a los sujetos de cuidado y establecer una relación de respeto y confianza. Por ello, la práctica se establece como un espacio que sensibiliza y fomenta el aprendizaje bioético, cuestiona al futuro profesional en su quehacer: "Creo que los

conocimientos que uno recolecta en la universidad le sirven a uno para realizar un trabajo bien hecho, pero no le sirve para entablar una relación efectiva con la otra persona [...], no solo enfocarse en el conocimiento de salud, sino ir un poco más allá y eso no se aprende con ir a clase, eso se aprende con convivir, con el respecto desde la casa" (Estudiante de Enfermería 1, intervención en grupo focal, 24 de octubre de 2017).

Concepto de bioética en el campo de la práctica profesional. bioética en enfermería
La práctica de la se fundamenta en general en los principios éticos y morales y en el respeto de los Derechos Humanos, los cuales guían las actuaciones de los profesionales en torno a los dilemas morales de base cultural, al brindar una pauta ética para el diálogo:

"Análisis sobre los interrogantes éticos que plantean los avances cientificos $y$ tecnológicos cuando involucra a seres humanos y formule recomendaciones que concilien la libertad de investigación con el respeto a la dignidad humana" (Documento nacional del Comité Intersectorial de Bioética de 2001). La práctica de enfermería se fundamenta en general en los Principios éticos y morales y en el respeto de los Derechos Humanos. (Documento nacional de reglamentación de la profesión de enfermería en Colombia de 1996)
Principios que orientan las prácticas de salud que para responder a las necesidades del sujeto de cuidado y su comunidad, incluyen el contexto, lo que exige en el estudiante competencias relacionadas con el cumplimiento de códigos deontológicos, disposición, compromiso y servicio.

Establece una visión más amplia de salud que reconoce la diversidad y cosmovisión del otro, por medio de la escucha y el contacto que, desde el contexto de práctica (en este caso la comunidad indígena misak), llama a la reflexión sobre la bioética en torno a la salud y su relación con el territorio que es donde la persona vive y convive en armonía con todo lo que le rodea: "Salud para nosotros va más allá de solo mirar la enfermedad; es pensar en el territorio [...] es lo que vivenciamos diariamente, lo que nos han enseñado. $Y$ a partir de esto nosotros podemos prevenir todo, por eso podemos hablar de salud mental, de salud sexual y reproductiva, de salud de todo"

(Profesional Hospital Mama Dominga, intervención en grupo focal, 10 de noviembre de 2017) punto de referencia y si yo

observo que es del $10 \%$ con respecto a un $90 \%$ (respecto al hacer, ¿eso significa que es menos importante? Eso genera un cuestionamiento supremamente grande" (Docente de Enfermería 9, intervención en grupo focal, 6 de diciembre de 2017).
La bioética, pese a que ha evolucionado en el discurso, dista en la práctica porque aún falta el análisis de cómo los elementos de respeto por la cosmovisión en torno al cuidado de la salud permean la toma de decisiones en su quehacer. Es por falta de esta reflexión que se encuentra en los estudiantes un cambio en sus comportamientos, según el contexto donde rotan, y los mínimos aprendizajes en torno a la bioética que logran adquirir quedan atrás. Quienes forman reconocen que no cuentan con bases epistémicas en torno a la bioética y reiteran que el currículo que orienta esta formación privilegia lo técnico y carece de procesos reflexivos. Aprender a convivir desde el contexto implica reconocer la participación de todos en el proceso de toma de decisiones en el acto de cuidado:

"Uno sabe que tiene que tratar a las personas desde todo su contexto y de forma holística [...] En [otro hospital] si vino es porque necesita ayuda y tiene que dejarse hacer lo que le hagan, en cambio acá, vino porque necesitaba ayuda, pero pues si eso va en contra de lo que consideran bien o mal, entonces pues se le respeta, se apunta asi: que 'se negó', pero 'se le respeta', en cambio allá no" (Estudiante
Aprender a convivir para comprender el cuidado de la salud como territorio. 


\begin{tabular}{|c|c|c|c|c|}
\hline $\begin{array}{l}\text { Interculturalidad para la } \\
\text { transformación de la } \\
\text { bioética en las prácticas } \\
\text { formativas en } \\
\text { enfermería. }\end{array}$ & 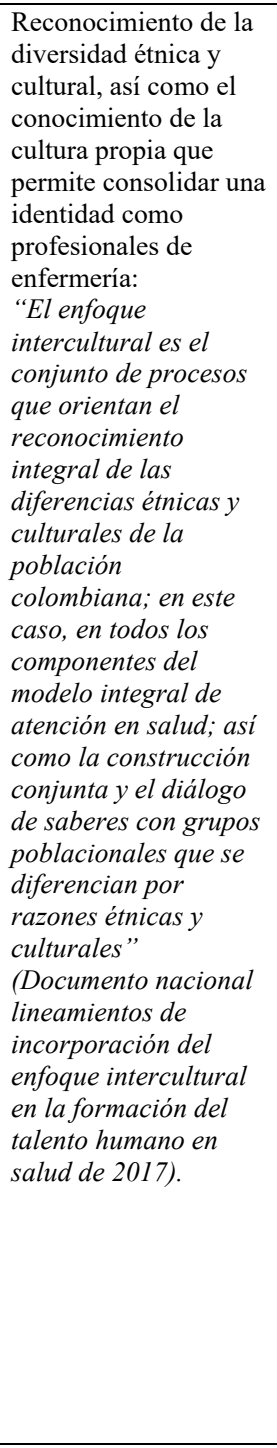 & $\begin{array}{l}\text { La interculturalidad se } \\
\text { establece como un puente } \\
\text { entre lo propio y lo } \\
\text { externo, que conlleva al } \\
\text { reconocimiento de la } \\
\text { diversidad, integración y } \\
\text { apropiación selectiva; es } \\
\text { decir, es la articulación de } \\
\text { la cosmovisión de las } \\
\text { personas en torno al } \\
\text { cuidado de la salud con los } \\
\text { conocimientos adquiridos } \\
\text { en la formación académica } \\
\text { de los estudiantes. Busca la } \\
\text { preservación cultural y el } \\
\text { respeto a la autonomía de } \\
\text { las personas al momento } \\
\text { de brindar el cuidado en } \\
\text { enfermería. Aquí la } \\
\text { comunicación es un eje } \\
\text { fundamental para } \\
\text { establecer una relación con } \\
\text { el otro, que no se limita al } \\
\text { lenguaje. Se requiere una } \\
\text { formación que incluya } \\
\text { otras visiones de salud que } \\
\text { permitan apertura a las } \\
\text { percepciones y } \\
\text { expectativas del otro. Así, } \\
\text { se demanda la articulación } \\
\text { de la universidad y el } \\
\text { contexto: } \\
\text { “Articular ambas culturas } \\
\text { de manera que se logre el } \\
\text { mayor beneficio para la } \\
\text { población sin tener que } \\
\text { llegar a choques culturales } \\
\text { sino a un acuerdo” } \\
\text { (Estudiante de Enfermería } \\
\text { 4, intervención en grupo } \\
\text { focal, 15 de noviembre de } \\
\text { 2017). }\end{array}$ & $\begin{array}{l}\text { La interculturalidad desde el } \\
\text { currículo se reduce al } \\
\text { desarrollo de competencias en } \\
\text { la relación y reconocimiento } \\
\text { del otro diverso, como una } \\
\text { estrategia de inclusión cuyo } \\
\text { propósito de formación se } \\
\text { construye para el contexto y } \\
\text { no desde el contexto, } \\
\text { continuando con la asimetría } \\
\text { de las relaciones entre la } \\
\text { universidad y el contexto de } \\
\text { práctica, como aquellas } \\
\text { propias del acto de cuidado. } \\
\text { Se demanda trascender hacia } \\
\text { una construcción de } \\
\text { interculturalidad que } \\
\text { considere a los seres humanos } \\
\text { con miradas distintas del } \\
\text { cuidado de la salud, y } \\
\text { contribuya a la } \\
\text { transformación de la bioética } \\
\text { en la práctica formativa, a } \\
\text { través de la escucha y } \\
\text { contacto temprano en } \\
\text { espacios de práctica } \\
\text { comunitarios concertados. } \\
\text { Así, las experiencias de } \\
\text { aprendizaje que se generan en } \\
\text { la práctica en contextos de } \\
\text { diversidad cultural, amplían la } \\
\text { concepción de cuidado y } \\
\text { posibilitan indagar sobre la } \\
\text { manera en la que otros } \\
\text { entienden el mundo, lo que } \\
\text { influye en su rol profesional: } \\
\text { "No es el lenguaje la barrera, } \\
\text { también es la actitud de los } \\
\text { profesionales de salud en la } \\
\text { atención, en la forma del } \\
\text { saludo, no los miran o no } \\
\text { responden" (Profesional } 6 \text { del } \\
\text { intervención en grupo focal, } \\
\text { 10 de noviembre de } 2017) \text {. }\end{array}$ & $\begin{array}{l}\text { Relaciones donde } \\
\text { no es el lenguaje } \\
\text { la barrera, es la } \\
\text { actitud. }\end{array}$ \\
\hline $\begin{array}{l}\text { Visión del cuidado en } \\
\text { enfermería para } \\
\text { transformar la bioética } \\
\text { en las prácticas } \\
\text { formativas en un } \\
\text { contexto intercultural. }\end{array}$ & $\begin{array}{l}\text { Se requiere de } \\
\text { formación ontológica } \\
\text { que reconozca el } \\
\text { carácter holístico e } \\
\text { irreductible del ser. Se } \\
\text { debe promover el } \\
\text { pensamiento crítico } \\
\text { para realizar un } \\
\text { cuidado } \\
\text { contextualizado y } \\
\text { transformador, un } \\
\text { cuidado responsable: } \\
\text { "El cuidado de salud } \\
\text { propia e intercultural } \\
\text { hace referencia a las } \\
\text { acciones en salud } \\
\text { propia y } \\
\text { complementaria que se } \\
\text { realizan con las } \\
\text { personas, las familias, } \\
\text { la comunidad y con el } \\
\text { territorio, que tiendan } \\
\text { a promover y proteger } \\
\text { la salud, y a preveniry } \\
\text { tratar la enfermedad } \\
\text { con el propósito de } \\
\text { promover, recuperar y }\end{array}$ & $\begin{array}{l}\text { El cuidado como esencia } \\
\text { de la enfermería requiere } \\
\text { considerar más que lo } \\
\text { físico de la persona, lo } \\
\text { emocional, la escucha y la } \\
\text { preocupación. Por tanto, el } \\
\text { rol del profesional de } \\
\text { enfermería necesita ir más } \\
\text { allá de la enfermedad en un } \\
\text { contexto que trasciende de } \\
\text { la asistencia para } \\
\text { considerar lo comunitario. } \\
\text { Esto con el fin de procurar } \\
\text { un cuidado integral, } \\
\text { incluyendo aspectos } \\
\text { metafísicos, lo que permite } \\
\text { considerar a las personas } \\
\text { como seres humanos } \\
\text { diversos y su salud como } \\
\text { producto de su interacción } \\
\text { armónica con el territorio: } \\
\text { "Para mi salud es ar- } \\
\text { monia; es decir, equilibrio } \\
\text { entre lo fisico, entro lo } \\
\text { emocional, lo psicológico, } \\
\text { lo cultural porque a veces } \\
\text { se choca y eso lo afecta a }\end{array}$ & $\begin{array}{l}\text { La visión epistémica y } \\
\text { ontológica de cuidado que } \\
\text { orienta la formación bioética } \\
\text { no es explícita en los } \\
\text { documentos institucionales. } \\
\text { Desde la voz de los actores se } \\
\text { infiere una visión de mundo } \\
\text { de reciprocidad que no solo } \\
\text { reclama la orientación } \\
\text { ontológica en el terreno } \\
\text { racional, sino de las } \\
\text { experiencias de aprendizaje } \\
\text { en la práctica formativa. De } \\
\text { esta manera, se propende por } \\
\text { una relación dialógica del } \\
\text { reconocimiento del otro, de su } \\
\text { saber y su responsabilidad en } \\
\text { torno al cuidado de la salud, } \\
\text { en la búsqueda de } \\
\text { denominadores comunes: } \\
\text { “Considero importante el } \\
\text { buen trato y amabilidad, así } \\
\text { usted no entienda [...]Si usted } \\
\text { desea aprender, se puede } \\
\text { aprender, pero con su } \\
\text { amabilidad es suficiente”, } \\
\text { (Sujeto de cuidado 9, }\end{array}$ & $\begin{array}{l}\text { Cuidado del } \\
\text { nosotros, } \\
\text { pensando en el } \\
\text { otro como uno } \\
\text { mismo. }\end{array}$ \\
\hline
\end{tabular}

de Enfermería 2, diario de

campo, 29 de septiembre de

formativas en

enfermería. conocimiento de la

iltura propia que

identidad como

profesionales de

conjunto de procesos

que orientan el

diferencias étnicas y

culturales de la

población

de saberes con grupos

poblacionales que se

diferencian por

culturales"

Documento nacional

enfoque intercultural

en la formación del

alento humano en

salud de 2017). continuando con la asimetría de las relaciones entre 1 versidad y el contexto de aquellas una construcción de ribuya a la nsformación de la bi tiva, spacios de práctica munitarios concertad diversidad cultural, amplían la epción de cuidado y profesiona:

es el lenguaje la barrera den "(Profesional $6 \mathrm{del}$

\section{La visión epistemica} experiencias de aprendizaje n la práctica formativa. D a, se propende "Considero importante el buen trato y amabilidad, as (Sujeto de cuidado 9 
Cultura de los Cuidados

mantener la armonía y uno; o sea como en todas

el equilibrio"

(Documento nacional

lineamientos de

incorporación del

enfoque intercultural

en la formación del

talento humano en

salud de 2017). las dimensiones que uno se

puede desarrollar"

(Estudiante de Enfermería

2 , intervención en grupo

focal, 15 de noviembre de 2017).

entrevista pers7onal, 4 de

noviembre de 2017).

"Mirarlos como personas!

[refiriéndose a los sujetos de

cuidado], no como un

paciente que necesita algo y

ya no más, sino mirar además

su entorno" (Estudiante de

Enfermería 2, intervención en

grupo focal, 15 de noviembre

de 2017).

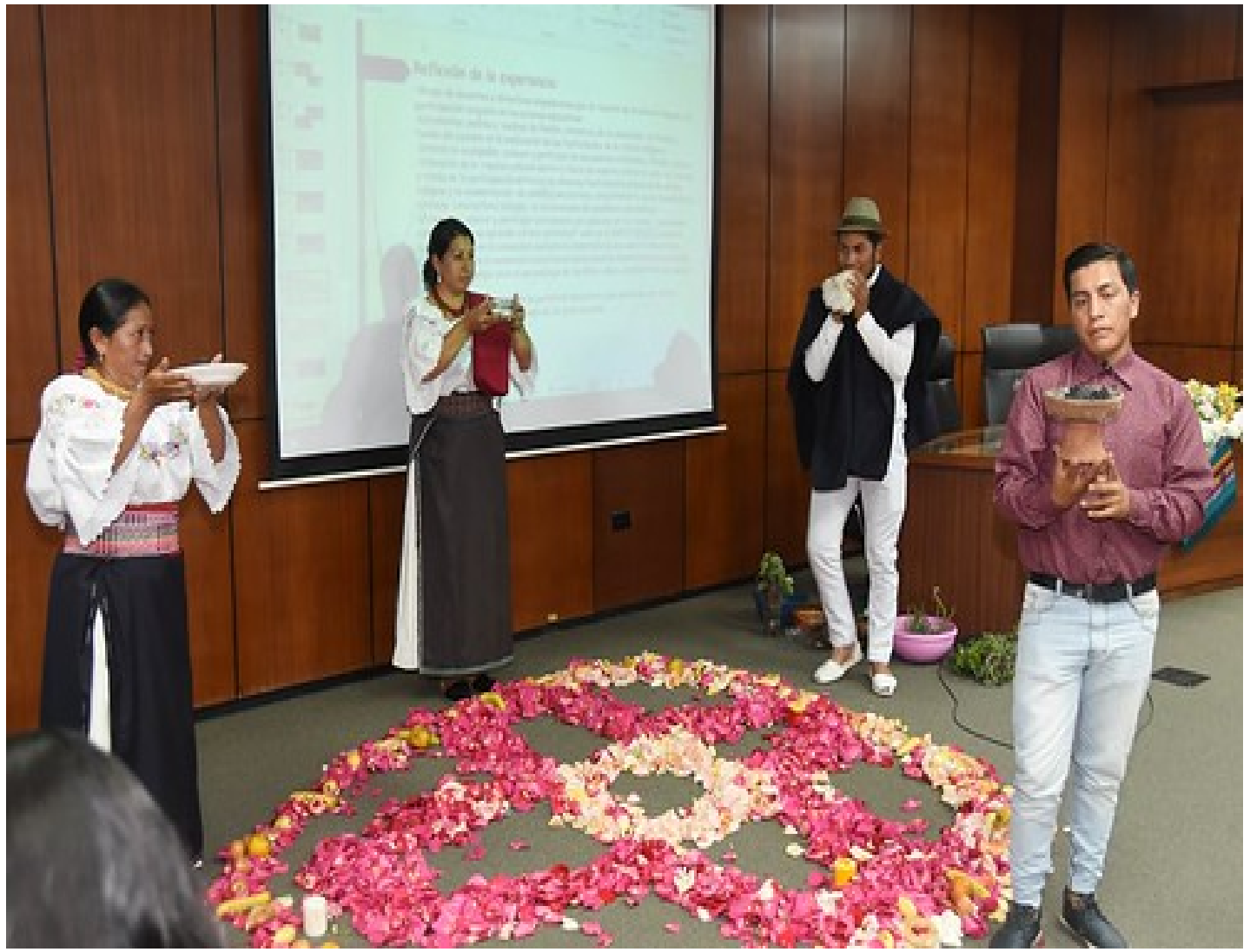

Fuente: CC BY-NC-SA 2.0 license. https://search.creativecommons.org/photos/eb238ec4f93e-4950-a4bf-e48bd42a990b 